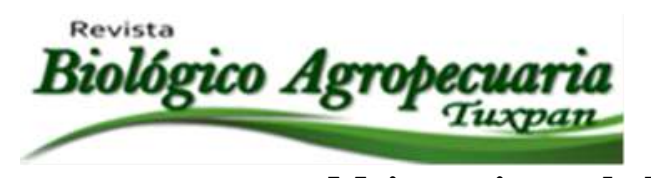

\title{
Mejoramiento de la imagen urbana en la ciudad de Chihuahua
}

Improvement of the urban image in the city of Chihuahua

\author{
Bustillos Duran Karla C. ${ }^{1}$. García Muñoz Silvia A. ${ }^{2 .,}$ Leyva Chávez Arwell N. ${ }^{2 凶}$., González Aldana \\ Ricardo A. ${ }^{2}$, Villalobos Pérez Elizabeth ${ }^{2}$., Ortega Rodríguez Anabel ${ }^{2}$. \\ ${ }^{1}$ Licenciatura en Desarrollo Territorial de la UACH. ${ }^{2}$ Facultad de Ciencias Agrotecnológicas, \\ Universidad Autónoma de Chihuahua Campus I, Chihuahua, Chihuahua., México. Tel. (614)4391844.
}

${ }^{\circledR}$ Autor para correspondencia: nleyva@uach.mx

Recibido: $15 / 04 / 2019$

Aceptado: 15/05/2019

\section{RESUMEN}

La historia de la ciudad es la de su espacio público. Para el caso de Chihuahua la imagen urbana vincula el espacio físico-cultural para la recreación de los habitantes, proporcionando un área verde y limpia acorde a la historia e identidad de la misma. Por necesidades de la población y el uso de suelo que se le da en cuestiones jurídicas a los espacios públicos, normalmente son utilizados como estacionamientos, inclusive los baldíos son convertidos en estacionamientos donde solo se va generando una plancha de concreto que no permite una oxigenación ni un entorno agradable a la población. Como función urbanística, innovar en estos lotes que no tienen uso dentro de la ciudad y que marcan una discontinuidad a la edificación termina por ser un limitante dentro de la imagen urbana y contaminación visual. Como función ecológica se brinda una oxigenación al entorno atenuando construcciones de valor paisajístico con orden y vegetación a una ciudad con identidad tomando en cuenta el espacio a nivel peatonal es decir contemplar la edad de la población para determinar el espacio más apto, el tamaño del lote y sus colindantes para la continuidad de la fachada proporcionando el orden e imagen a una ciudad con espacio suficientes para estacionamientos, espacios verdes, áreas de recreo, áreas de descanso o la unión de estos espacios. Se considera la ciudad y su diseño a nivel peatonal, así como la escala, edades, necesidades de la población y su entorno, con la finalidad de encontrar un espacio público abierto acorde a vii estas necesidades que se presenten; dando prioridad a zonas de valor paisajístico en conjunto con el reglamento de imagen urbana y paisaje del municipio de Chihuahua. Recreación, imagen urbana, patrimonio histórico y cultural son temas primordiales de la ciudad de Chihuahua para efectos del tema de estudio, se consideraron avenidas que cruzaran por la mitad de la ciudad, ya que son vías que comunican de norte a sur a la población, así como transporte externo a la zona urbana, con la finalidad de cuantificar, medir y dimensionar los espacios, lotes o baldíos desperdiciados y en des uso dentro de la mancha urbana, dando un total de 92 zonas en diferentes colonias adjuntas a la Av. Tecnológico, Av. Carlos Pacheco, Bldv. Fuentes Mares y así como las avenidas colindantes a las principales.

Palabras clave: Físico-Cultural, Recreación. 


\begin{abstract}
The citizen public space is not a residuary place between streets and buildings. It is not an empty one considered "public" just for legal reasons, neither a "specialized" space, which one has to be visited like a museum or a concert (Borja, 2000). The history of the city is the one about its public space. For the case of Chihuahua, the urban image links the physical space-culture for the recreation of the people, providing a clean green zone proper to its history and identity. By necessities of the people and the uses of the ground that is given into legal matters to the public places, normally are used as parking lots, even the wastelands are turned into parking lots where only concrete spots are being produced that doesn't allow any oxygenation, neither an enjoyable environment to the people. As urban function, to innovate in this batches that have no use within the city and mark a discontinuity to the edification ending by being a limit in the urban image and visual pollution. As ecological function, is brought an oxygenation to the environment weaken some monuments with view nature, with order and vegetation to a city with identity considering the space into a pedestrian level, that is to say, to view the age of the people to determinate the most suitable space, the size of the batch and its adjacent places for the continuity of the facade providing the order and image to a city with enough space for parking lots, green zones, rest areas, playtime zones or the mixing of this places. The city is considered and it's design into a pedestrian level, as well as scale, ages, necessities of the people and it's environment, with the goal of find one open public space according to the necessities that show up, giving priority to places of view value altogether with the rules of the urban image and view of the Township of Chihuahua. ix Recreation, urban image, historic and cultural heritage are primordial topics of Chihuahua City for the topic of studying, are considered avenues that will through the city because they are ways that links from north to south of the population, as well as external transportation to the urban zone to quantify, measure and to size the space, batches or wasted wastelands with no use, giving a result of ninety two zones in different suburbs attached to Technologic Avenue, Carlos Pacheco Avenue, Fuentes Mares Boulevard and as well as the near avenues to the main ones.
\end{abstract}

Keywords: Physical-Cultural, Recreation.

\section{INTRODUCCIÓN}

Perahia, (2017) de la Universidad Federal de Rio Grande do Sul dice que el espacio público es el que da identidad y carácter a la ciudad, el que permite reconocerla y vivirla en sus sitios urbanos: naturales, culturales y patrimoniales.

Es la necesidad de las pequeñas o grandes ciudades para vincular el espacio físico-cultural para la recreación con sus habitantes, proporcionando el interés debido, con un fácil acceso a todos, agregando la imagen urbana que proporciona un área verde y limpia acorde al entorno donde se encuentra.

Los motivos por los cuales la ciudad de Chihuahua no cuenta con infraestructura adecuada para el tema de recreación e imagen urbana pueden ser variados, el vandalismo, desinterés por parte de la población y de las autoridades, dificultades de movilidad, así como económicas.

Por esto mismo se debe considerar el diseño urbano a nivel peatonal, así como la escala, edades y necesidades del entorno, reforzando la 
calidad del diseño de los espacios públicos para tener un uso mixto del suelo con una mezcla social y elementos funcionales que propicien la cohesión social dando prioridad a las zonas, vialidades, sitios o inmuebles de valor paisajístico considerados así por su carácter patrimonial dentro del reglamento de imagen urbana y paisaje del municipio de Chihuahua. Tomando en cuenta las avenidas, bulevares y periféricos considerados patrimonio por su historia cultural y valor paisajístico.

En el caso y por imagen urbana los espacios verdes serian lo primordial, sea plaza o parque, incorporar el concepto de espacio libre con vegetación predominando la importancia a nivel peatonal en esta área de descanso, paseo y recreo en horas de ocio.

La necesidad que se tiene en la ciudad de Chihuahua de recreación en un espacio abierto, necesidad que probablemente como habitante empleo-hogar dormitorio, no se toma en cuenta o no se le da la importancia que tiene, de la misma manera hacer notar que las plazas no son exclusivas de niños o adultos mayores, si no que todas las personas en distintas edades tienes derecho y necesidad de tener un momento de recreo.

Sin dejar el enfoque a todas las edades que se encuentran en la zona, un lugar donde el porcentaje de población económicamente activa tenga oportunidad del disfrute de un espacio abierto, no precisamente considerando que tengan más derecho, pero en aspecto económico son los que aportan, y socialmente necesitan de momentos de ocio.

\section{MATERIALES Y MÉTODOS}

En el presente trabajo se analizan diferentes esquemas de manera sustentable de las avenidas que cruzan por la mitad de la ciudad de Chihuahua, el tema abordado ubica principalmente espacios dentro de estas vías de comunicación que no tienen uso, ya sean viviendas abandonadas, edificaciones en tapias, así como terrenos baldíos; siendo así, se toma como principal la imagen urbana que puede brindar un confort a la sociedad que transite por las vías primarias, así como de la necesidad de cohesión social en cada colonia, enfocando factores acordes a la población aledaña.

Entre los fenómenos que intervienen sobre el espacio natural es el ser humano el que modifica el espacio para mejoras político-ambientales y socio-culturales, el espacio tiene una lógica interna que brinda sentido de pertenencia a sus habitantes, por ello la gestión en beneficio político-social enfrenta variables como el clima, uso y permisos del suelo, ya que los escases que presenta la capital en espacios públicos abiertos vegetados genera poca convivencia, más impacto ecológico así como una densificación poco eficiente; Por este análisis se toman las siguientes avenidas de la ciudad de Chihuahua:

- Unión carreta libre y cuota AhumadaChihuahua- Av. Tecnológico: $4.41 \mathrm{Km}$

- Av. Tecnológico -Av. Colon :11.89 Km,

- Av. Juárez - Blvd. Fuentes Mares: 11.2 Km

- Bldv. Fuentes Mares- Unión periférico R. Almada $4.1 \mathrm{Km}$.

Con un total de $26.2 \mathrm{Km}$ de recorrido desde $\mathrm{Km}$ 4 carretera Juárez hasta término del periférico $\mathrm{R}$. Almada. 


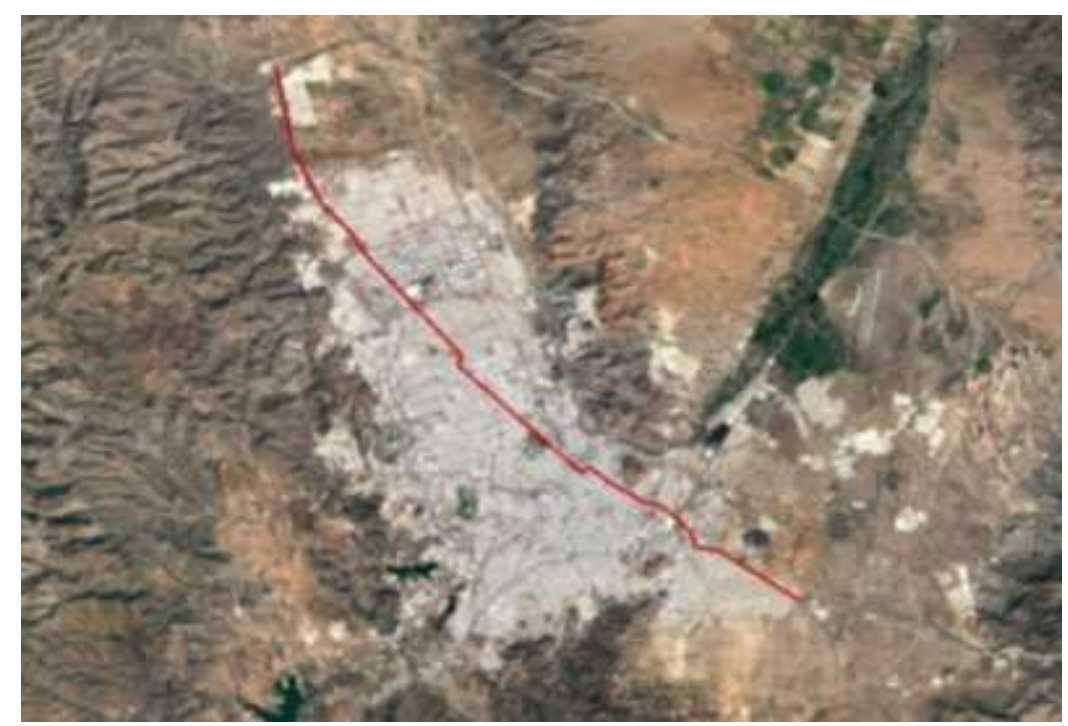

Figura 1. Delimitación del área de estudio

La identificación y análisis de los baldíos fueron realizados con ArcGis, Google earth pro.

Todas las zonas fueron digitalizadas desde fotografías aéreas georreferenciadas para medir, ubicar y delimitar cada baldío que se encontraba a lado de las vialidades, así como el DENUE para seccionar por edades de población con radio definido dependiendo el tamaño del lote, para tomar el número de población beneficiada como principal

\section{RESULTADOS}

- 37 lotes para población adulta, entre 15-59 años de edad.

- 17 niños de 0-14 años de edad.

- 25 sin población aledaña, por uso de suelo industrial o comercial.

- 31 adultos mayores de 64 y más.

- 14 personas con discapacidad,

Las zonas donde los adultos mayores predominan son en la colonia Las Granjas y Zaragoza, Alta Vista, Santo Niño y Villa Juárez.
La población adulta se encuentra dispersa a lo largo de las vialidades analizadas.

Para el caso de la población con alguna discapacidad se encuentra en las colonias: Lealtad 2, Obrera de Ávalos, Villa Juárez, Ávalos.

Algunos baldíos se encuentran mixtos, ya sea población adulto mayor, discapacidad y adultos, o niños y adultos, las primeras colonias de la ciudad de Chihuahua se encuentran dentro de las mencionadas con personas discapacitadas y adulto mayor.

\section{CONCLUSIÓN}

El análisis que se llevó acabo, arrojo información principalmente socio-ambiental, donde se percata de la falta de áreas verdes dentro la ciudad de Chihuahua, en este caso en las avenidas que cruzan por la mitad de la ciudad.

Es importante mencionar la perspectiva de la imagen urbana donde la ciudad es deficiente, ya 
que las edificaciones no se encuentran acorde a la historia y entorno local, también la falta de lugares de recreo y ocio para las diferentes edades de la población.

Dado que los beneficios sociales y ambientales por conservar y revitalizar áreas verdes dependen de la accesibilidad a la que se tiene, por tanto, tener la plaza, parque o área verde en común genera la cohesión social que se busca en cualquier sociedad.

Además de la importancia de los valores estéticos, las áreas verdes contribuyen a la depuración del aire. Generan microclimas y contribuyen a disminuir la temperatura en el entorno ubicado.

También permiten tener la visión peatonal que se va perdiendo en las grandes urbes, creando ciudades para automóviles, manejando y abordando problemáticas de tránsito vehicular y no peatonal, elevando niveles de estrés y bajo desempeño laboral, creando una insuficiente conciencia por la cultura ambiental.
Para el tema de imagen urbana, la investigación arroja los lineamientos que se deben cumplir dependiendo el uso de suelo, la vocación, la cultura, el entorno y el paisaje, y la importancia en que los habitantes perciban la ciudad, los espacios contrastantes ayudan a las personas a recordar y tener el sentido de pertenencia, donde la población exprese valores y buenas conductas disminuyendo la delincuencia.

Dicho esto, la dinámica de la población de la ciudad de Chihuahua delimitará los espacios disponibles para áreas verdes, creando zonas pensadas en la población aledaña y los habitantes que pasen por las avenidas principales mencionadas.

Generando el conocimiento y conciencia responsable con el análisis urbanístico que permite un paisaje adecuado, disminuyendo niveles de contaminación y brindando áreas de esparcimiento. 


\section{ANEXOS}

Ejemplo de los espacios delimitados y seccionados dependiendo la edad de la población.
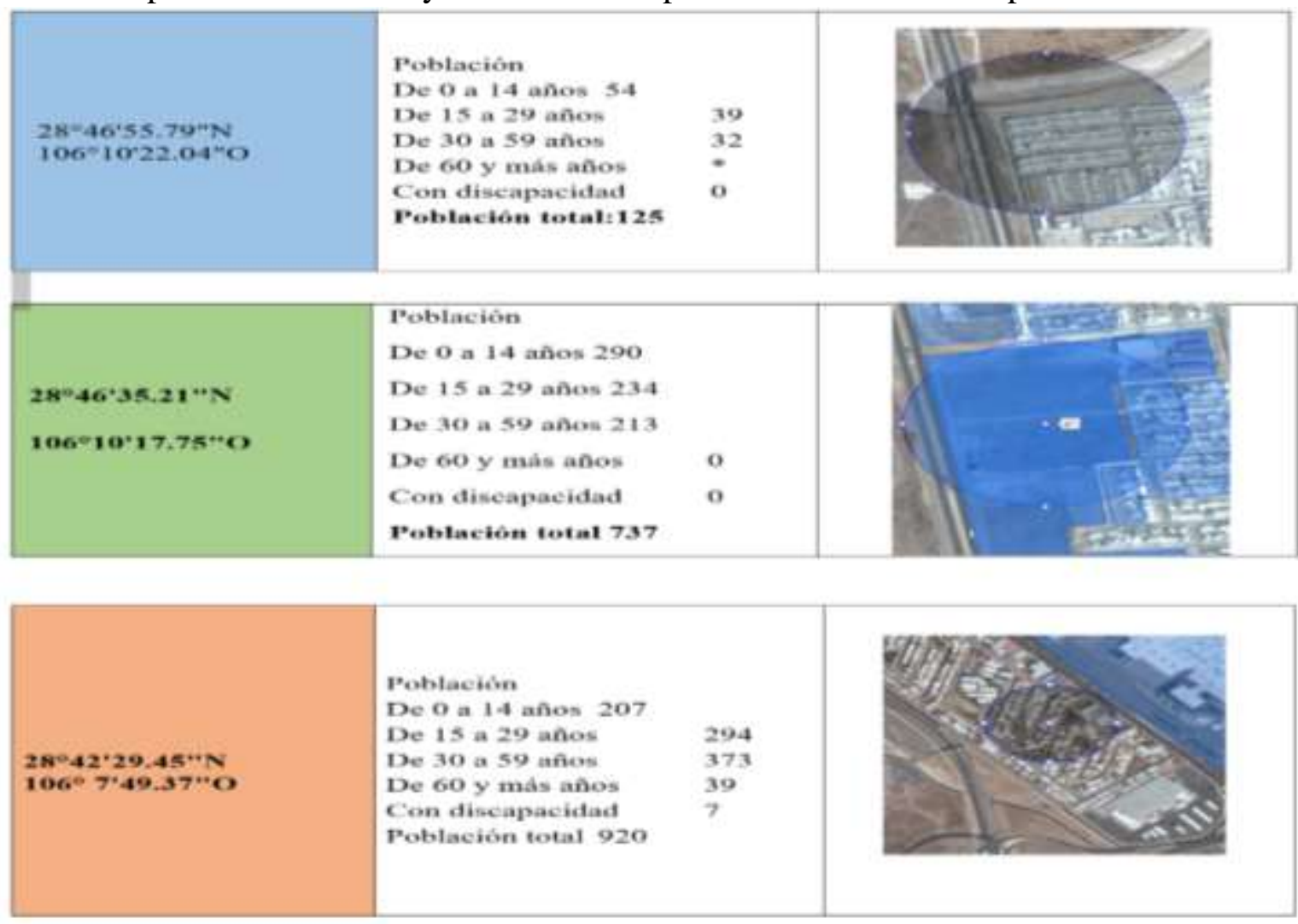

\section{LITERATURA CITADA}

Aires, G. B. (2016). Propuestas para adolescentes. Obtenido de https://turismo.buenosaires.gob.ar/es/art icle/propuestas-para-adolescentes

Borja, J. (2000). El espacio público, ciudad y ciudadanía. Obtenido de server2.docfoc.us/uploads/z2015/11/18/ 7cgehhzy6s/ef5e25dfbfd1ae6214f17c9a af00b3af.pdf

Chihuahua, G. D. (5 de octumbre de 2013). Reglamento de desarrollo urbano sostenible del municipio de Chihuahua.

Chihuahua, P. O. (2001). Reglamento de construcciones y normas técnicas para el municipio. Obtenido

de http://www.smie.org.mx/layout/reglame ntos-construccion/chihuahuareglamento-construccion-municipal1.pdf

Conapo. (2012). Dinamica demografica 19902010 y proyecciones de poblacion 2010 2030. Obtenido de http://www.conapo.gob.mx/work/model s/conapo/proyecciones/cuadernos/08_cu adernillo_chihuahua.pdf

Implan. (2009). Plan de desarrollo urbano de la ciudad de Chihuahua: visión 2040. Obtenido de http://www.implanchihuahua.gob.mx/pd u2040/pdf/diagnostico_movilidad.pdf 
Bustillos et al., 2019

Madrid, U. C. (2015). De plaza en plaza. Unicef. (2012). Derechos de la niñez. Obtenido Obtenido de de

https://deplazaenplaza.wordpress.com/2 https://www.unicef.org/mexico/spanish/ 015/05/26/espacio-publico-como-un17054.htm derecho/

Copyright (c) 2019 Karla C. Bustillos Durán, Silvia A. G arcia Muñoz, Arwell N. L eyva Chávez, Ricardo A. G onzález Aldana, Elizabeth Villalobos Pérez y Anabel Ortega Rodriguez

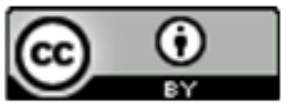

E ste tex to está protegido por una licencia Creative Commons 4.0 .

Usted es libre para Compartir — copiar y redistribuir el material en cualquier medio o formato- y Adaptar el documento - remezclar, transformar y crear a partir del material- para cualquier propósito, incluso para fines com erciales, siempre que cumpla la condición de:

Atribución: Usted debe dar crédito a la obra original de manera adecuada, proporcionar un enlace a la licencia, e in dicar si se han realizado cambios. Puede hacerlo en cualquier forma razonable, pero no de forma tal que sugiera que tiene el apoyo del licenciante o lo recibe por el uso que hace de la obra.

Resumendelicencia - Textocompletodelalicencia 